\title{
Sturzprävention für geriatrische Handpatienten
}

\author{
Frank Blömker
}

Gründe für eine Immobilität im Alter können die Folgen einer medikamentös induzierten Sturzsymptomatik sein. Aber auch fehlende Balance und Sturzangst sind mögliche Ursachen von mangelnder Bewegung. Wie kann das Sturzrisiko bei älteren Menschen erfasst werden und welche Maßnahmen sind präventiv möglich?

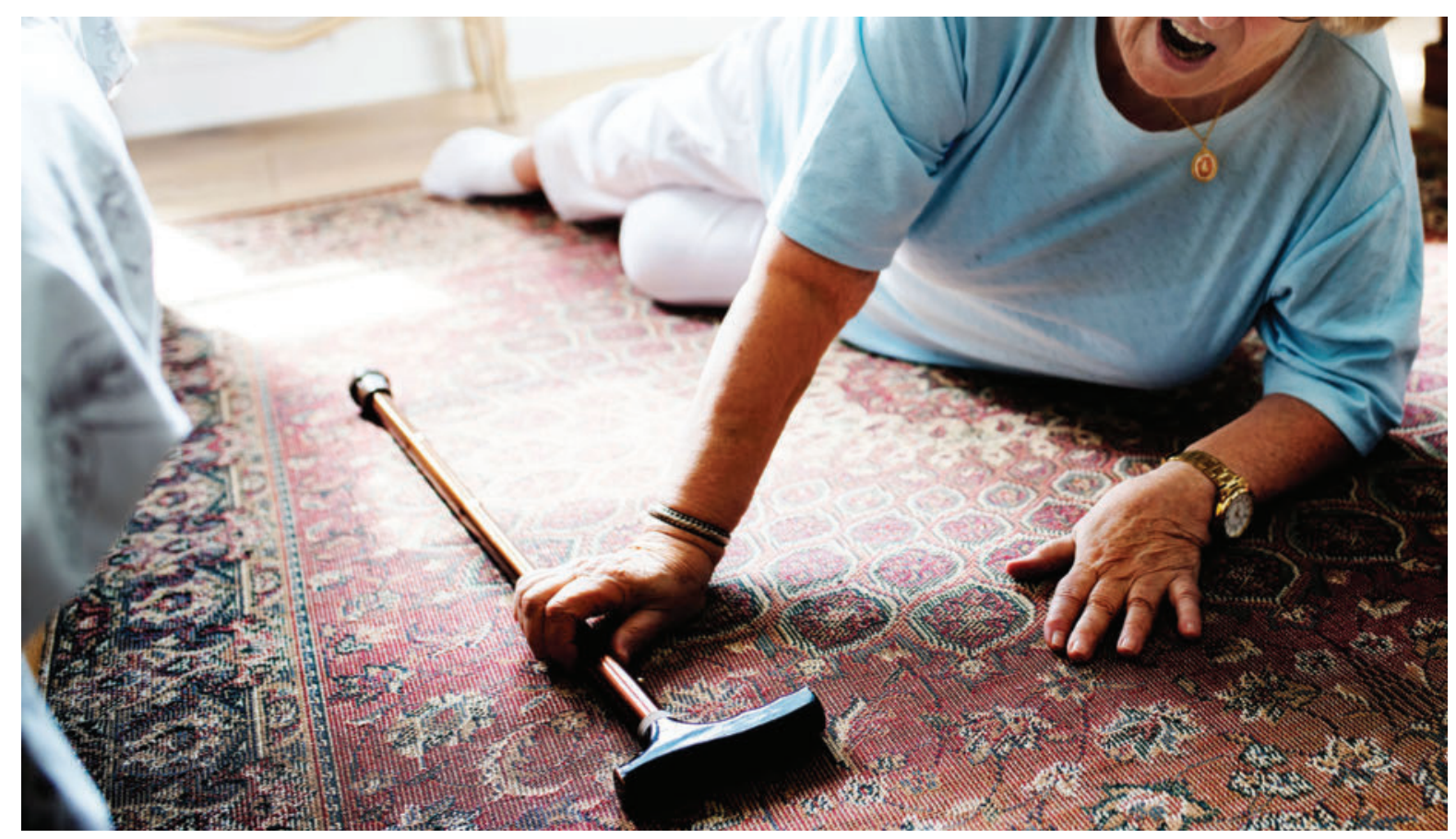

Stürzen im Alter hat weitreichende Folgen. (Quelle: Rawpixel.com/shutterstock.com; Symbolbild)

Derzeit wird das Angebot an präventiven Maßnahmen für Seniorinnen und Senioren stark erweitert. So hält zum Beispiel die Bundeszentrale für gesundheitliche Aufklärung (BZgA) umfassende Programme vor, die verschiedene Themen des Eigentrainings anschaulich und seniorengerecht aufarbeitet. Thematisiert werden beispielsweise Sturzangst, Krafttraining und Balancetraining. Die Broschüren zur Sturzprävention können Interessierte im Internet unter www.bzga.de kostenfrei bestellen oder als PDF herunterladen.

\section{Erfassung des Sturzrisikos}

Zunächst wird die medikamentös induzierte Sturzsymptomatik ärztlich beurteilt und ggf. behoben. Gründe können eine fehlende Dosisanpassung, die Einnahme von Antihypertensiva, additive Effekte, Neuroleptika und Tranquilizer sowie Schlafmedikationen sein.

Spätestens nach jedem Sturz sollte zudem eine kritische Analyse sturzgefährdender Wohnumstände vorgenommen werden. Diese führen der Sozialmedizinische Dienst oder Angehörige durch. Insbesondere die hohe Inzidenz von Sturzereignissen zeitnah nach Ortswechseln (z. B. Residenzoder Krankenhauseinweisung) oder beim nächtlichen Toilettengang muss zu Ablaufänderungen führen. In der frühen postoperativen Nachbehandlung ist außerdem eine funktionelle Analyse motorischer Schwachstellen anzuraten, sofern sie nicht bereits in einer geriatrischen Versorgungseinheit durchgeführt wurde. Sie sollte möglichst 
unter Einbeziehung pflegender Dritter erfolgen. Umfassende Assessments, wie beispielsweise der Timed „Up and Go“-Test oder der Mini-Mental-Status-Test, geben eine gute Übersicht über funktionelle und kognitive Probleme.

\section{Maßnahmen zur Sturzprävention}

In der Anpassung der Wohnumgebung sind Haltegriffe und besondere Beleuchtungen sowie ein angepasstes Mobiliar notwendig. In Krankenhäusern ist darüber hinaus die Einbindung von „Lotsen“ und betreuenden Angehörigen für diese Patientengruppe wichtig. Krankenhauslotsen kennen die Einrichtung und begleiten Patienten zu ihren Behandlungen oder der Station. Seit 1996 ermöglicht zudem das sog. Bielefelder Modell, Senioren ein selbstbestimmtes Wohnen mit Versorgungssicherheit, ohne dass dabei eine Betreuungspauschale anfällt. Inhalte des Konzepts sind u.a. barrierefreie Wohnungen, Servicestützpunkte vor Ort und Begleitung von Aktivitäten, Hobbys und Kultur [1].

Nicht selten empfehlen Ärzte ihren Patienten mit rezidivierenden Sturzereignissen prophylaktisch die Anwendung von Hüftprotektoren. Neben dem meist fragwürdigen Effekt im Hinblick auf die Prophylaxe von Niedrigenergietraumen ist die Patienten-Compliance dafür allerdings ausgesprochen schlecht.

Bewährt hat sich ein individuell angepasstes Balancetraining, das der Patient ritualisiert in die Tagesplanung einbezieht. Als Übungen für zu Hause bieten sich Aktivitäten des täglichen Lebens an, die durch eine Gleichgewichts-, Koordinations- oder Haltungsübung ergänzt werden. Beispielsweise kann die sturzgefährdete Person während des morgendlichen Zähneputzens auf einem Bein stehen [2].

Additiv kann dem motorisch-funktionellen Defizit durch Hirnleistungstraining begegnet werden. Essenziell ist bei allen Maßnahmen die Einbindung der Angehörigen. Frühe Therapieeinheiten sollten Brücken in die ambulante Versorgung bauen. Eine enge Rückkopplung zwischen Arzt und Therapeut ist empfehlenswert.

Im Hinblick auf die Orthetik ist darauf zu achten, dass verordnete Hilfsmittel sich sehr stark voneinander unterscheiden und häufig nicht der individuelle Bedarf, sondern letztlich der Marktpreis entscheidet.

Für die Gruppe Hochbetagter sind Orthesen zu fordern, die bei guter Passform leicht anzubringen sind. Außerdem sollten sie bei axialer Stauchung so viel Druckentlastung wie möglich bewirken. Die Orthesen müssen überwacht und gegebenenfalls modifiziert werden.
Immobilisierende Orthesen sollten nur so kurz wie möglich getragen werden. Geriatrietypische Veränderungen wie fortgeschrittene Polyarthrosen müssen dabei berücksichtigt werden. Hier sind individuell konfektionierte Lösungen zu wählen. Für die Benutzung von Gehhilfen und Rollatoren sind anatomische Griffformen aus Weichgummimaterial zu fordern. Hier sind am Markt zahlreiche Versorgungsmöglichkeiten gegeben.

\section{Ausblick}

Gegenwärtig steht eine Reihe von Maßnahmen aus dem Bereich „smart home“ an, die das Gefährdungspotenzial von Seniorinnen und Senioren verbessern helfen. Ebenso sind Entwicklungen im Bereich „Exoskelett“ möglicherweise geeignet, bestehende Defizite schnell und sicher zu überbrücken. Durch ein Exoskelett kann beispielsweise ein bestehendes motorisches Defizit durch eine mechanische Koppelung an ein patientenindividuell angepasstes Modell kompensiert werden. Die Prävention sollte ein wesentlicher Baustein eines Behandlungsalgorithmus sein und so die Ganzheitlichkeit des Therapieansatzes-zum Beispiel auch durch die Einbindung von Angehörigen - verdeutlichen.

\section{Autorinnen / Autoren}

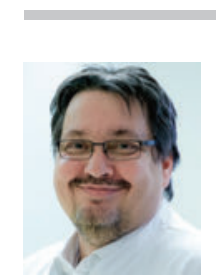

\begin{abstract}
Frank Blömker
Facharzt für Orthopädie und Unfallchirurgie und seit $2011 \mathrm{im}$ medizinischen Leitungsteam der Akademie für Handrehabilitation. Er ist Chefarzt der Klinik für Unfallchirurgie an der Katholischen Hospitalvereinigung WeserEgge. Davor war er lange Chefarzt der Abteilung für Unfall - und Handchirurgie und Sporttraumatologie am AGAPLESION Ev.Bathildiskrankenhaus in Bad Pyrmont.
\end{abstract}

\section{Korrespondenzadresse}

\section{Frank Blömker}

St. Vincenz Hospital

Danziger Str. 17

33034 Brakel

E-Mail: F.Bloemker@khwe.de

\section{Literatur}

[1] https://bgw-bielefeld.de/wp-content/uploads/DownloadsBGW/ 04_Infos_und_Flyer/Bielefelder_Model_web.pdf

[2] https://www.bzga.de/infomaterialien/gesundheit-aelterermenschen/gleichgewicht-und-kraft-einfuehrung-in-diesturzpraevention/ 